\title{
A Study on the Risk Factors and Protective Factors the Depression of Dementia Elderly's Primary caregiver in South Korea
}

\author{
SUNWON PARK · JAE YOP KIM
}

\begin{abstract}
\section{Purpose}

The purpose of this study is to examine the effects of symptoms level of dementia elderly on the primary caregiver's depression. In addition, moderating effect of social support was examined.
\end{abstract}

\section{Methods}

Based on the results, the necessity for intervention in the level of social welfare as a way to mitigate primary caregiver's depression was suggested. In order to accomplish these purposes, a total of 197 who are spouse and adult children as primary caregivers of dementia elderly using day care facilities or services in South Korea. Date were analyzed by frequency analysis and descriptive statistics, regression model analysis with SPSS 18.0.

\section{Results}

In case of analysis results, the mean value was reported 0.9 out of four point about primary caregiver's depression and the mean value of social support was reported 3.34 out of five point. And besides, the analysis result of dementia elderly's symptoms showed that prevalence of depression/dysphoria were $62.2 \%$, prevalence of aberrant motor were $61.3 \%$, prevalence of apathy/indifference were $56.6 \%$. Crucial findings are as follows: the symptoms level of dementia elderly was significantly associated with the primary caregiver's depression. At the same time, social support significantly influenced lower level of the primary caregiver's depression. 


\section{Conclusion}

While, in the relationship between the symptoms level of dementia elderly and the primary caregiver's depression, social support has a moderation effect by important protection factor. From these findings, the necessities to provide the care service for dementia elderly to help improve symptoms of dementia as well as the policy and service to manage the mental health of the social network as primary caregiver were suggested. Also, the necessities to provide the therapy program to improve the relationship with medical team, friends, and family members were suggested.

\section{Key Words: Dementia Elderly, Primary caregiver, Depression, Dementia Symptoms.}

